\title{
Egg allergy and MMR vaccine: New recommendations from the National Advisory Committee on Immunization
}

$\mathrm{T}^{\mathrm{h}}$ he fourth edition of the Canadian Immunization Guide (1993) recommends that "persons who have a history of anaphylactic hypersensitivity to hens' eggs (urticaria, swelling of the mouth and throat, difficulty in breathing or hypotension) should not be given measles vaccine except under special precautions". The precautions outlined include skin testing with diluted vaccine and graded-challenge vaccination if the skin test is positive. Results of several recent studies have questioned such a cautious approach. The National Advisory Committee on Immunization (NACI) has reviewed all available data and revised its guidelines accordingly. The following revised guidelines are a major departure from the previously published recommendations. They will appear in the next edition of the Canadian Immunization Guide.

A measles-rubella combination vaccine (Mo-Ru Viraten Berna, Switzerland) recently licensed in Canada contains no avian proteins and therefore can be used without regard to egg allergy.

Vaccines that contain small quantities of egg protein can cause hypersensitivity reactions in some people with egg allergy. Adverse reactions are more likely with vaccines, such as yellow fever and influenza vaccines, that are grown in embryonated eggs. In contrast, measles and mumps vaccine viruses, which are most widely used in Canada, are grown in

This article appeared in the Can Commun Dis Rep 1996;22-14:113-5. Reproduced with permission.

All material presented in Pediatric Infectious Disease Notes has been reviewed and approved by the chairperson, Canadian Paediatric Society Board and representative members of the Canadian Paediatric Society Committee on Infectious Diseases and Immunization

Correspondence: Infectious Diseases and Immunization Committee, Canadian Paediatric Society, 401 Smyth Road, Ottawa, Ontario K1H 8L1. Telephone 613-737-2728, fax 613-737-2794 chick-embryo cell culture. Even after extensive purification, final vaccine products may contain trace quantities of avian proteins resembling proteins present in hens' eggs $(1,2)$.

Anaphylaxis after administering measles-containing vaccines is rare and has been reported in individuals with anaphylactic hypersensitivity to eggs as well as those with no history of egg allergy. In some of these instances, allergy to neomycin $(3,4)$ or gelatin (5) was hypothesized but, in most cases, no allergen was identified (6-8).

Because of rare anaphylactic reactions after measles-containing vaccines, NACI had recommended that measles-mumps-rubella (MMR) skin testing be performed in individuals with anaphylactic hypersensitivity to eggs. Recent studies have raised questions about the usefulness of and a rationale for these recommendations. These studies have reported uneventful routine MMR immunization in egg-allergic individuals (8-11) and in those with positive MMR skin tests (12). Others have reported occasional adverse reactions despite the use of MMR skin testing and graded challenge (13-15). In a Canadian study, 500 egg-allergic children including 33 with respiratory distress associated with egg ingestion were safely immunized; skin testing was abandoned after the first 120 children because of its lack of predictiveness (16). Most recently, 54 children with egg allergy, including three with positive MMR skin tests, were routinely immunized without problem (17). In reviewing the literature, these investigators calculated that over 1200 individuals with egg allergy have been assessed for measles immunization. None of the 284 children with egg allergy confirmed by blinded food challenge had any problem with routine measles immunization (95\% CI $99.0 \%$ to $100 \%$ ).

Routine immunization was tolerated in all of 1209 children with positive skin tests for egg allergy (95\% CI 99.75\% to 100\%) and in 1225 of 1227 (99.84\%) children with histories of egg allergy ( $95 \% \mathrm{Cl} 99.41 \%$ to $99.98 \%$ ). In addition, 38 anaphylac- 
tic reactions after measles immunization have been reported in the literature in individuals without a history of egg allergy; MMR skin tests were positive in only four of the nine (44.4\%) individuals tested (17).

\section{RECOMMENDATIONS}

In view of the cumulative data indicating the safety of measles immunization in individuals with a history of anaphylactic hypersensitivity to hens' eggs and the lack of evidence of the predictive value of MMR skin testing, NACI has revised its recommendations for MMR immunization of individuals allergic to eggs as follows:

- As previously recommended by NACI, all immunizations should be administered by persons capable of managing vaccine-associated adverse reactions such as anaphylaxis and should take place in appropriate facilities.

- Egg allergy is not a contraindication to immunization with MMR. In individuals with histories of anaphylactic hypersensitivity to hens' eggs (urticaria, swelling of the mouth and throat, difficulty breathing or hypotension), measles immunization can be administered in the routine manner without prior skin testing. However, immunization should take place where adequate

\section{REFERENCES}

1. Aukrust L, Almeland TL, Refsum D, et al. Severe hypersensitivity or intolerance reactions to measles vaccine in six children. Allergy 1980;35:581-7.

2. Herman JJ, Radin R, Schneiderman R. Allergic reactions to measles (rubeola) vaccine in patients hypersensitive to egg protein. J Pediatr 1983;102:196-9.

3. Elliman D, Dhanraj B. Safe MMR vaccination despite neomycin allergy. Lancet 1991;337:365.

4. Kwittken PL, Rosen S, Sweinberg SK. MMR vaccine and neomycin allergy. Am J Dis Child 1993;147:128-9.

5. Kelso JM, Jones RT, Yunginger JW. Anaphylaxis to measles, mumps, and rubella vaccine mediated by IgE to gelatin. J Allergy Clin Immunol 1993;91:867-72.

6. Van Asperen PP, McEnlery J, Kemp AS. Immediate reactions following live attenuated measles vaccine. Med J Aust 1981;2:330-1.

7. McEwan J. Early-onset reaction after measles vaccination Further Australian reports. Med J Aust 1983;2:503-5.

8. Kemp A, Van Asperen P, Mukhi A. Measles immunization in children with clinical reactions to egg protein. Am J Dis Child 1990;144:33-5.

9. Bruno G, Giampietro PG, Grandolfo ME, et al. Safety of measles immunisation in children with IgE-mediated egg allergy. Lancet 1990;335:739.

10. Beck SA, Williams LW, Shirrell A, et al. Egg hypersensitivity and measles-mumps-rubella vaccine administration. Pediatrics 1991;88:913-7.

11. Fasano MB, Wood RA, Cooke SK, et al. Egg hypersensitivity and adverse reactions to measles, mumps, and rubella vaccine. J Pediatr 1992;120:878-81.

12. Aickin R, Hill D, Kemp A. Measles immunisation in children with allergy to egg. BMJ 1994;309:223-5.

13. Lavi S, Zimmerman B, Koren G, et al. Administration of measles, mumps, and rubella virus vaccine (live) to egg-allergic children. JAMA 1990;263:269-71. facilities are available to manage anaphylaxis. Persons at risk should be observed for 30 mins after immunization for any signs of allergic reaction. No special precautions are necessary for children with minor egg hypersensitivity, which permits uneventful ingestion of small quantities of egg, or when measles-rubella vaccine free of avian proteins is used. No special measures are necessary in children who have never been fed eggs before MMR immunization. Prior egg ingestion should not be a prerequisite for MMR immunization.

- Measles vaccine (or MMR) is contraindicated in individuals with a previous anaphylactic reaction to a measles-containing vaccine. If there is a compelling reason to re-immunize an individual who has had a prior anaphylactic reaction to measles vaccine, MMR skin testing and graded challenge in an appropriately equipped facility can be considered. However, the possibility of a hypersensitivity reaction to the MMR skin test or during the graded challenge must be considered.

- $\quad$ Surveillance for post measles vaccine anaphylaxis should be improved and prospective studies should be initiated to better define the risk in individuals with egg allergy.

14. Puvvada L, Silverman B, Bassett C, et al. Systemic reactions to measles-mumps-rubella vaccine skin testing. Pediatrics 1993;91:835-6.

15. Trotter AC, Stone BD, Laszlo DJ, et al. Measles, mumps, rubella vaccine administration in egg-sensitive children; systemic reactions during vaccine desensitization. Ann Allergy 1994;72:25-8.

16. Freigang B, Jadavji TP, Freigang DW. Lack of adverse reactions to measles, mumps, and rubella vaccine in egg-allergic children. Ann Allergy 1994;73:486-8.

17. James JM, Burks AW, Roberson PK, et al. Safe administration of the measles vaccine to children allergic to eggs. $\mathrm{N}$ Engl J Med $1995 ; 332: 1262-6$.

National Advisory Committee on Immunization (NACl) Members: D Scheifele (chairman); J Spika (executive secretary); N Armstrong; (adminstrative secretary); F Aoki, P DeWals, E Ford-Jones, I Gemmill, S Halperin, B Law, $M$ Naus, Y Robert, $B$ Ward. Liaison Members: LCdr D Carpenter, Department of National Defense; A Carter, Canadian Medical Association;

T Freeman, College of Family Practice of Canada; $S$ Hadler, Centers for Disease Control and Prevention; D MacPherson, Committee to Advise on Tropical Medicine and Travel; V Marchessault, Canadian Paediatric Society; I Walters, Advisory Committee on Epidemiology.

Ex-officio representatives: $P$ Duclos, Laboratory Centre for Disease Control; C Mustard, Medical Services Branch, Health Canada; P Palkonyay, Drugs Directorate, Health Canada 


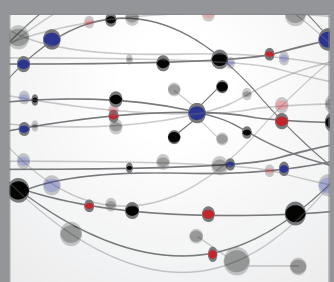

The Scientific World Journal
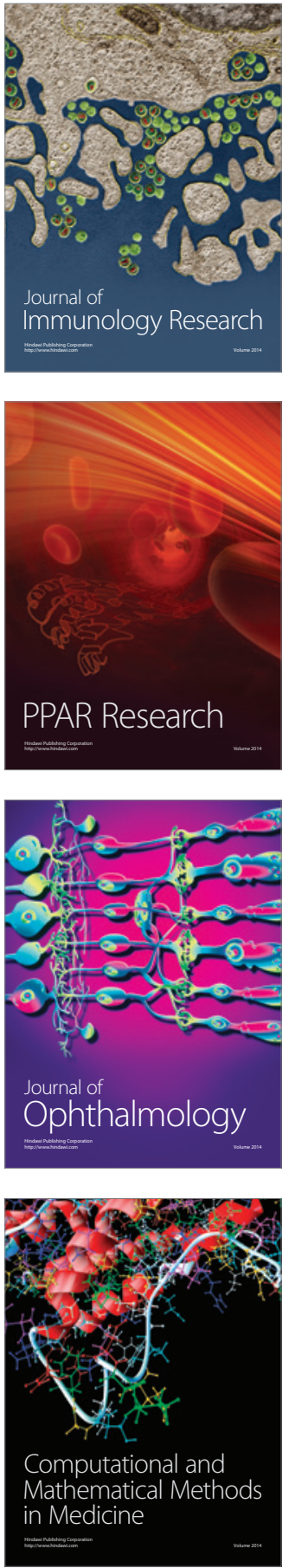

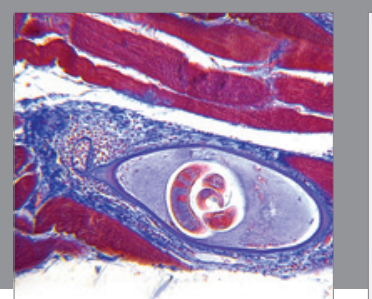

Gastroenterology Research and Practice

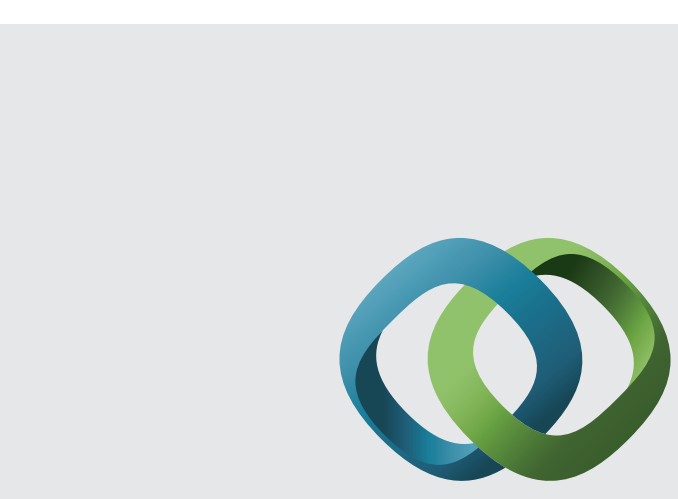

\section{Hindawi}

Submit your manuscripts at

http://www.hindawi.com
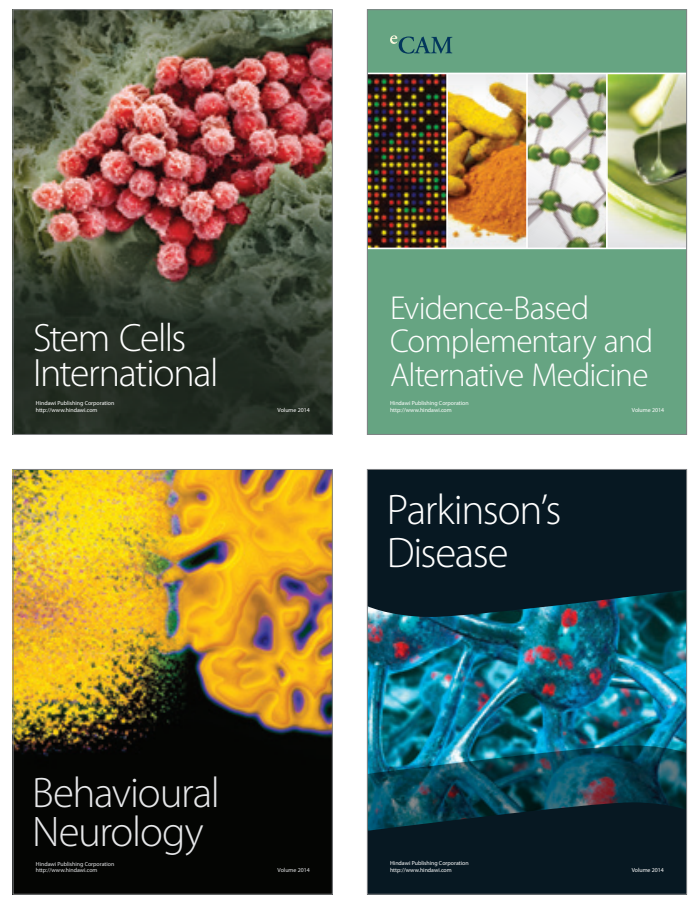
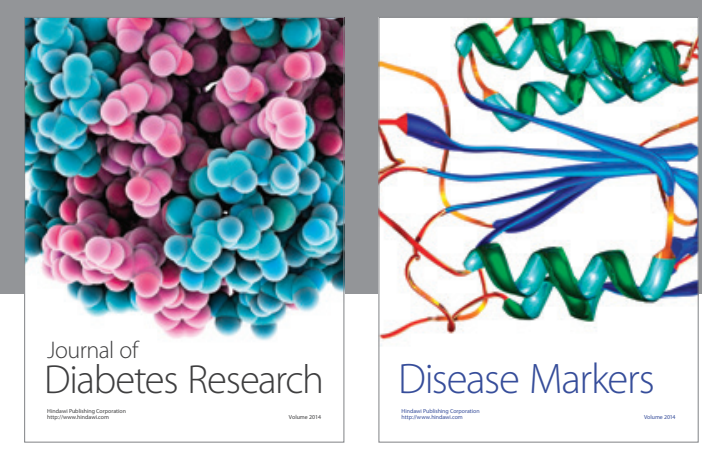

Disease Markers
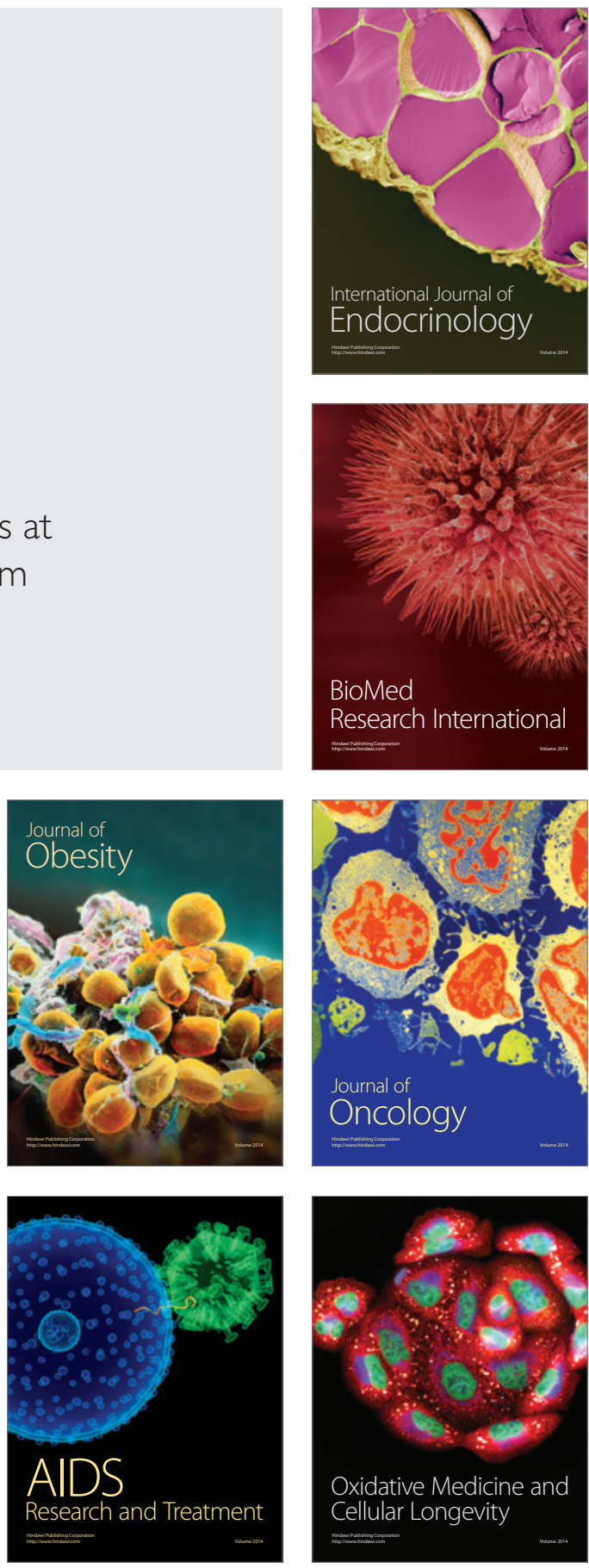